\title{
ASSOCIATION BETWEEN SKELETAL MUSCLE METABOLOMICS PROFILE AND CARDIORESPIRATORY FITNESS IN YOUNG MEN.
}

\author{
Lucas de Marchi Silva (IC), Alex Castro, Renata Garbellini Duft, Marina Lívia Ferreira Venturini, André Luís \\ Lugnani de Andrade, Cláudia Regina Cavaglieri, Mara Patrícia Traina Chacon-Mikahil (PQ).
}

\begin{abstract}
High cardiorespiratory fitness levels (CRF) are strongly associated with reduction of risk of all-cause and cardiovascular disease mortality. CRF can be assessed by maximal oxygen uptake (VO2MAX) or maximal power output (MPO). Skeletal muscle is the main organ responsible for peripheral adaptations related to CRF improvement. In this sense, metabolomics has been pointing out as a potential tool to identify muscular metabolic signature in response to exercise and different phenotypes. Therefore, this study aimed to investigate metabolites in human skeletal muscle associated with CRF. Participated in this study 70 sedentary young men (18-30 years old). Vastus lateralis muscle biopsies were collected at fasting state (12 h). After 72 h, CRF was assessed by MPO, obtained during a maximal incremental test performed until voluntary exhaustion in cycle ergometer, followed by re-test $48 \mathrm{~h}$ later. Skeletal muscle samples were analysed through metabolomics (H1 NMR spectroscopy). Associations of baseline metabolites with MPO were explored via three analytical strategies (three levels of evidence): (1) correlations with gains in MPO; (2) significant difference between groups of and Low CRF and High CRF; and (3) metabolite contribution to significant pathways related to MPO. The significance level was set up at $\mathrm{P}<0.01$ or false discovery rate of 0.1 . From 70 identified metabolites, only Formate and NADP + were supported by the three levels of evidence. Intrinsic CRF levels are positively associated with higher baseline metabolites levels (Formate and NADP+) and pathways indicative mainly of the energy metabolism.
\end{abstract}

\section{Key words:}

Maximal Power Output, Metabolomics, Skeletal Muscle.

\section{Introduction}

High cardiorespiratory fitness levels (CRF) are strongly associated with reduction of risk of all cause and cardiovascular disease mortality. Recently metabolomics has been used to map and create metabolic profiles in different biological samples. Metabolic profile analysis has the potential to identify new biomarkers related to CRF. To investigate biomarkers associated to CRF can contribute to health monitoring and clarify the biology of this complex phenotype. In this sense, assuming skeletal muscle as the main organ responsible for peripheral adaptations related to CRF improvement, this study aimed to investigate metabolites in human skeletal muscle associated with CRF.

\section{Results and Discussion}

Participated in this study 70 sedentary young men (age: $23 \pm 3$ years old; body mass index: $24 \pm 3 \mathrm{~kg} . \mathrm{m}-2)$. Vastus lateralis muscle biopsies were collected at fasting state (12 h). After 72 h, CRF was assessed by MPO obtained during maximal incremental test performed until voluntary exhaustion in cycle ergometer, followed by re- test $48 \mathrm{~h}$ later. Skeletal muscle samples were analysed through metabolomics (H1 NMR spectroscopy). Only baseline metabolites supported by three levels of evidence were associated with MPO and discussed. This approach included: (1) correlations with gains in MPO (Pearson correlation coefficient of $r \geq 0.2$; (2) significant difference between groups of individuals with high and low CRF (based on first and third tertiles of MPO distribution); and (3) metabolite contribution to significant pathways related to MPO. The significance level was set up at $P<0.01$ or false discovery rate of 0.1 . If the confidence intervals for the effect size (ES - Cohen's d) of comparisons did not cross zero, the difference was considered significant.

From 70 identified metabolites, only Formate and NADP+ were supported by the three levels of evidence. These metabolites were included in 5 significant pathways associated to CRF (Formate: pyruvate metabolism, nitrogen metabolism, and methane metabolism; NADP+: nicotinate and nicotinamide metabolism and glutathione metabolism; Figure 1A). Both Formate and NADP+ demonstrated significant higher baseline levels for High CRF compared to Low- CRF groups (Figure 1B).
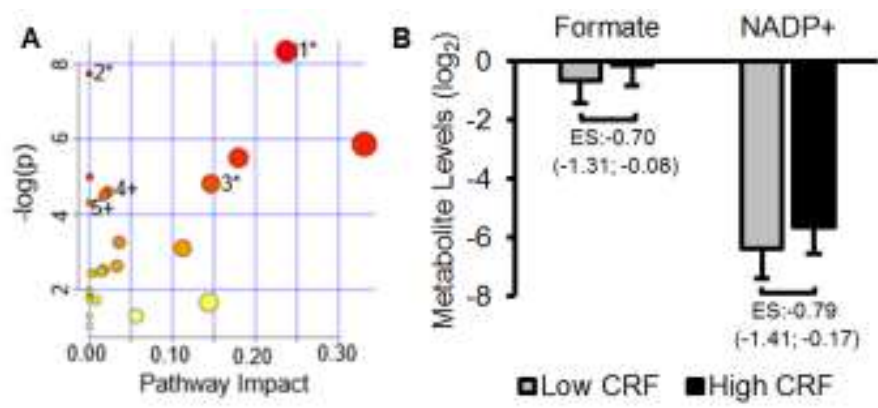

Figure 1. (A) Metabolic pathways related to CRF. 1: pyruvate metabolism; 2: nitrogen metabolism; and 3 : methane metabolism; 4: glutathione metabolism; 5: Nicotinate and nicotinamide metabolism; * Formate has been included; ${ }^{+} \mathrm{NADP}+$ has been included. (B) Significant differences between Low CRF e High CRF. SD: Standard Deviation. ES: Effect Size.

\section{Conclusions}

Intrinsic CRF levels are positively associated with higher baseline metabolites levels (Formate and NADP+) and pathways indicative mainly of energy metabolism.

\section{Acknowledgement}

FAPESP, PIBIC, CNPq, FAEPEX, FISEX-FEF- UNICAMP and LNBio-CNPEM. 